\title{
On the corresponding states law of the Yukawa fluid
}

\author{
Pedro Orea and Yurko Duda \\ Programa de Ingeniería Molecular, Instituto Mexicano del Petróleo, Eje Central 152, 07r30 México D.F., México,
}

\begin{abstract}
We have analyzed the currently available simulation results, as well as performed some additional Monte Carlo simulation for the hard-core attractive Yukawa fluid in order to study its corresponding state behavior. We show that the values of reduced surface tension map onto the master curve, and a universal equation of state can be obtained in the wide range of the attractive Yukawa tail length after a certain re-scaling of the number density. Some comparisons with other nonconformal potentials are presented and discussed.
\end{abstract}

PACS numbers:

\section{INTRODUCTION}

The investigation of colloid agglomeration, and phase stability has been a subject of long-standing theoretical and practical interest $1,2,3,4,5$. It is well accepted that the range of the effective interaction between particles in different solvents determines the shape and location of the phase boundaries of colloidal dispersions 4,5 . From a theoretical point of view, the phase transition in colloidsolvent solution is analogous to that of the single component gas-liquid system. Thus, the most general approach is to assume a form for the interparticle effective pair potential. One of the most common selection is the Yukawa potential,

$$
u(r)= \begin{cases}\infty, & \text { if } r<\sigma \\ -\epsilon \sigma \exp [-\kappa(r-\sigma)] / r, & \text { if } \sigma \leq r\end{cases}
$$

where parameters $\epsilon$ and $\kappa$ define the depth and range of the potential, respectively; $\sigma$ is a particle diameter, which is usually taken as a unit length, $\sigma=1$.

The range of attraction in a pair potential cannot be changed for ordinary fluids, but it can be adjusted in colloid-solvent systems by adding, for instance, a nonadsorbing polymers, ions, or other solutes ${ }^{1,2,4}$. Therefore, upon varying the range of the interaction in the Yukawa potential (1), one can repoduce the behavior of some real systems.

The phase diagram and interfacial properties of the hard-core attractive Yukawa (HAY) fluid have been investigated by means of the theoretical tools of the liquid-state statistical mechanics $5,6,7,8,9,10$, and computer simulations $11,12,13,14,15$. The simulation for measuring any thermophysical property or phase equilibria for HAY fluid is, in most cases, time consuming and expensive due to long-range character of the potential. That is why HAY fluid with long attraction tails have been recently studied by applying an Ewald sum 15,16.

On the other hand, it is well-known that in some cases the application of the corresponding states (CS) law is a very useful approach to avoid extensive computer calculations as well as experimental measurings $17,18,19,20,21,22,23$. According to the CS law the substances have unified equation of state

$$
P_{R}=F\left(T_{R}, \rho_{R}\right)
$$

in reduced variables of pressure, $P_{R}=P / P_{c}$, temperature, $T_{R}=T / T_{c}$, and number density, $\rho_{R}=\rho / \rho_{c}$; the subscript $c$ denotes critical values, and $T \equiv k_{B} T / \epsilon$, as usual. In Eq. $2 F$ is a universal, but complicated, function. However, it is well-known that the CS approach is valid only for conformal pair potentials, and Yukawa potential is not one of them, because its range of attraction varies independently of the hard-core radius $\sigma$. Taking this into account, some researchers have applied the so-called extended CS law for a nonconformal potential, i.e. when Eq. 2 involves a third parameter ${ }^{20}$. Besides, there have been speculations based on experimental observations and computer simulations for various nonconformal model potentials that the gas-liquid coexistence curves may follow an extended CS theory, if they are plotted in terms of the reduced second virial coefficient, $B_{2}^{*}=B_{2}\left(T_{c}\right) / B_{2}^{H S}$ (where $B_{2}^{H S}$ is the second virial coefficient of hard sphere fluid), and the reduced density $\rho_{R}{ }^{23}$. Such considerations were based on the assumption that second virial coefficient remains practically constant at the critical point.

However, more recently it has been shown, that $B_{2}^{*}$ varies with the range of attractions, in contrast to the common belief that it remains practically constant for different pair potentials 9.24 . The results of $B_{2}^{*}$ for the HAY fluid from $\kappa=7$ to $\kappa=0.5$ are presented in Table 1. Indeed, its values are far from being constant.

\section{CS LAW APPLICATION ANALYSIS}

In order to investigate the applicability of the CS law in the case of HAY fluid, we have analyzed the available simulation data 11,13,14,15 and performed some additional Monte Carlo (MC) simulation for relatively long attraction tail, $\kappa=1.8$ and $\kappa=1.5$. Our simulation study has been performed in the canonical ensemble as described in Refs $11,13,14$. For the phase diagram definition and surface tension calculations, MC simulations were performed on a parallelepiped cell with dimensions $L_{x}=L_{y}=12$, and $L_{z} \geq 45$. The pressure of the supercritical HAY 
fluid was calculated in a cubic simulation cell, $L_{x}=12$. In each simulation, the value of cut-off radius $r_{c u t}$ of the potential (11) was selected to be 5 . Such value of $r_{c u t}$ as well as the system size have been proved to be sufficient for obtaining accurate results 13,14. Our new results together with the previously published results, are given in Tables 1 and 2.

The critical parameters for the HAY fluid were calculated by using the rectiliniear diameter law and the universal value of critical exponent $\beta=0.325$. The critical pressures were estimated on the base of ClausiusClapeyron equation $\underline{21}$, and together with other critical parameters are given in Table 3.

In fig. 1 we present the dependence of the critical HAY fluid density $\rho_{c}$ and pressure $P_{c}$, on the inverse critical temperature $T_{c}^{-1}$ and critical temperature $T_{c}$, respectively. Besides our results for the attractive range varying from $\kappa=7$ to $\kappa=1.5$, in the left panel of the figure we have used the critical data for $\kappa=0.5$, and 1.0 estimated from the recent work of Caillol et al.$^{15}$. All the presented data indicate a clear linear dependencies described by the following equations:

$$
\begin{gathered}
P_{c}=0.0228+0.0742 T_{c}, \\
\rho_{c}=0.2534+0.071 \frac{1}{T_{c}} .
\end{gathered}
$$

Such liner behavior leads to the constant value of the critical compressibility factor, $Z_{c}=P_{c} /\left(\rho_{c} T_{c}\right)=$ $0.3 \pm 0.01$ in the range of $\kappa$ considered (see also Table 3 ). This value of $Z_{c}$ coincides surprisingly well with its universal value reported in the literature for different real substances 17 . Actually, in some cases, this value is being used to estimate the critical pressure ${ }^{22}$.

In the left part of the Fig.1 we present also the results of the so-called self-consistent Ornstein-Zernike approximation (SCOZA) reported in Ref ${ }^{6.15}$. As seen, there is a linear dependence of $\rho_{c}$ vs $1 / T_{c}$, too. However, the prediction of the theory deteriorates at the short attractive Yukawa-tails, $\kappa \geq 4$. This tendency has been discussed in Ref. $\underline{6}$, where the authors have addressed some tentative reasons of the SCOZA inaccuracy when the range of the pair potential narrows. Namely, it was supposed that modification of the closure condition equation may be needed.

We have also performed preliminary analysis of the available critical data for the fluids with (i) Sutherlandlike attractive pair interactions, which vary like $r^{-3-t}$ (with $t=3,1$, and $0.1^{25}$ ), and (ii) Mie $n-6(7<n<32$ ) potential21. In both cases, there is also a linear dependence between $\rho_{c}$ and $1 / T_{c}$, although it is slightly different from the relation (44). For the case of the Mie fluid, a linear function $P_{c}=P_{c}\left(T_{c}\right)$ is presented in the right side of Fig.1. It is interesting to note, that there is a region where critical parameters of HAY and Mie fluids almost coincide.

On the other hand, the widely used square-well pair potential shows, according to SCOZA predictions ${ }^{26}$, com- pletely nonlinear behavior of the $\rho_{c}=\rho_{c}\left(T_{c}^{-1}\right)$, as can be seen in Fig.1.

If $\rho^{L}$ and $\rho^{G}$ denote the liquid and the vapor in mutual equilibrium at the temperature $T$, respectively, then according to CS law one should expect $\rho_{R}^{L, G}$ to be universal functions of $T_{R}$. In Fig.2 the liquid-vapor coexistence curves scaled by the critical values of density and temperature are presented for different $\kappa$ 's. It is seen that most of the points lie on or near a single curve. More interestingly, the rectiliniar diameter line, which is defined as 17 ,

$$
\frac{\rho^{L}+\rho^{G}}{2 \rho_{c}}=1+A\left(1-\frac{T}{T_{c}}\right)
$$

and formed by the simulation results, is very close to the argon diameter line, with $A=3 / 4$, depicted as dashed line in Fig. 2.

Since the surface tension, $\gamma$ does not have a reduced analogue like $T_{R}$ or $P_{R}$, because $\gamma_{R}=\gamma / \gamma_{c}$ is not defined $\left(\gamma_{c}=0\right)$, the CS law has been successfully applied for some substances $\frac{17,20}{}$ assuming the universality of $\gamma_{r}=\gamma /\left(\rho_{c}^{2 / 3} T_{c}\right)$ with respect to $T_{R}$. As we show in Fig. 3, all the surface tension simulation data for the HAY fluid with $\kappa=7,5,1.8$ and 1.5 map onto the same master curve if presented in units of $\gamma_{r}$. Such CS behavior of the reduced surface tension may answer the questions about the precision of some recent approximate density functional theories $27,28,29$, which predict unexplained nonlinar trends of $\gamma_{r}=\gamma_{r}\left(T_{R}\right)$ function.

The surface tension calculations have been performed through the pressure tensor ${ }^{13}$, therefore, it was natural to suppose that $\rho_{r}=\rho / \rho_{c}^{2 / 3}$ (instead of $\rho_{R}$ ) should be used in the CS law application for the calculation of the reduced pressure, $P_{R}$. We have verified such hypothesis and the results are presented in Fig.4 and Table 2. As seen, there is a perfect coincidence of $P_{R}$ for HAY fluid with $\kappa=1.8$ and 7 , at the three reduced temperatures considered.

\section{CONCLUSIONS}

This work presents the detailed analysis of the relations between the critical parameters of HAY fluid with variable range of attraction. Such analysis is based on the recently reported accurate simulation ${ }^{11,13,14,15}$ and integral equation data ${ }^{6.15}$. We show that there is a linear relationship between critical pressure and critical temperature, as well as, critical density and inverse critical temperature for HAY fluid for various interaction ranges. We have also detected such linearity for the bulk critical parameters of $n-6^{21}$ and Sutherland-like $e^{25}$ potentials with varying attractive tail length. We have shown that reduced pressure and surface tension of HAY fluid obeys the corresponding state law in the range $7<\kappa<1.5$ if the re-scaling critical number fluid density $\rho_{c}$ is re- 
placed by $\rho_{c}^{2 / 3}$. Finally, our findings permit us to conclude that phase diagram, pressure and surface tensions of the HAY fluid can be successfully obtained applying the properly modified corresponding state theory. Thus, we introduce a new criteria for the estimation and improving of the theoretical approaches $5,6,7,8,9,27,28,29$, i.e. now the predictive accuracy of different theories can be verified by applying of CS law. It is very probable, that other fluids interacting via an attractive nonconformal potential $21,22,25$ can be studied with the same CS ap- proach. To verify such hypothesis additional accurate simulations are desirable.

\section{ACKNOWLEDGMENTS}

We gratefully acknowledge the financial support of the Instituto Mexicano del Petróleo under the Projects D.31519/D.00406.
1 E. Zaccarelli, J.Phys.: Condens. Matt. 19, 323101(2007).

2 D. F. Rosenbaum, A. Kulkarni, S. Ramakrishnan, and C. F. Zukoski, J. Chem. Phys. 111, 9882 (1999).

3 M. Chávez-Páez, P.González-Mozuelos, M. MedinaNoyola, and J.M. Méndez-Alcaraz, J. Chem. Phys. 119, 7461 (2003).

${ }^{4}$ G. Cassin, Y. Duda, M. Holovko, J. P. Badiali, and M. Pileni, J. Chem. Phys. 107, 2683 (1997).

${ }^{5}$ F. W. Tavares and J. M. Prausnitz, Colloid Polym. Sci. 282, 620 (2004).

${ }^{6}$ G. Foffi, G. D. McCullagh, A. Lawlor, E. Zaccarelli, K. A. Dawson, F. Sciortino, P. Tartaglia, D. Pini, and G. Stell, Phys. Rev. E 65, 031407 (2002); C. Caccamo, G. Pellicane, D. Costa, D. Pini, and G. Stell, Phys. Rev. E 60, 5533 (1999).

7 J. H. Herrera, H. Ruiz-Estrada, and L. Blum, J. Chem. Phys. 104, 6327 (1996); D. M. Duh and L. Mier-y-Terrán, Mol. Phys. 90, 373 (1997).

${ }^{8}$ Y. V. Kalyuzhnyi, C. McCabe, E. Whitebay, and P. T. Cummings, J. Chem. Phys. 121, 8128 (2004).

${ }^{9}$ D. Fu, Y. Li, and J. Wu, Phys. Rev. E 68, 011403 (2003).

10 M. Hasegawa, J. Chem. Phys. 108, 208 (1998).

11 E. Lomba and N. G. Almarza, J. Chem. Phys. 100, 8367 (1994).

12 M. Dijkstra, Phys. Rev. E 66, 021402 (2002).

13 Y. Duda, A. Romero-Martinez, and P. Orea, J. Chem. Phys. 126, 224510 (2007).

14 U. F. Galicia-Pimentel, J. López-Lemus, and P. Orea, Fluid Phase Equil. (in press) (2008).

15 J. M. Caillol, F. Lo Verso, E. Schöll-Paschinger, and J. J. Weis, Mol. Phys. 105, 1813 (2007).

16 M. Mazars, J. Chem. Phys. 126, 056101 (2007).

17 E. A. Guggenheim, J. Chem. Phys. 13, 253 (1945).

18 G. Foffi and F. Sciortino, Phys. Rev. E 74, 050401 (2006).

19 E. M. Alfel, V.S. Vorob'ev, and G. A. Martynov, J. Phys. Chem. B 110, 8474 (2006).

20 A. J. Castellanos, G. Urbina-Villalba, and M. GarciaSucre, J. Phys. Chem. 108, 5951 (2004).

21 H. Okumura and F. Yonezawa, J. Chem. Phys. 113, 9162 (2000).

22 D. O. Dunikov, S. P. Malyshenko, and V. V. Zhakhovski, J. Chem. Phys. 115, 6623 (2001).

23 M. G. Noro and D. Frenkel, J. Chem. Phys. 113, 2941 (2000).

24 R. Lopez-Rendon, Y. Reyes, and P.Orea, J. Chem. Phys. 125, 084508 (2006).

25 P. J. Camp and G. N. Patey, J. Chem. Phys. 114, 399 (2001).

${ }^{26}$ E. Schöll-Paschinger, A. L. Benavides, and R. Castaeda-
Priego, J. Chem. Phys. 123, 234513 (2005).

27 V. C. Weiss and W. Schröer, Int. J. Thermod. 28, 506 (2007).

28 D. Fu and J. Wu, Mol. Phys. 102, 1479 (2004).

29 G. J. Gloor, F. J. Blas, E. M. del Río, E. de Miguel, and G. Jackson, Fluid Phase Equil. 194-197, 521 (2002). 
TABLE I: Canonical Monte Carlo data for the phase equilibrium and surface tension of the HAY fluid with $\kappa=1.5$ and 1.8 .

\begin{tabular}{cccc}
\hline \hline$T$ & $\rho_{L}$ & $\rho_{G}$ & $\gamma$ \\
\hline \multicolumn{5}{c}{$\kappa=1.5$} \\
1.10 & 0.730 & 0.0207 & $0.760_{20}$ \\
1.15 & 0.702 & 0.0290 & $0.633_{15}$ \\
1.20 & 0.665 & 0.0415 & $0.515_{20}$ \\
1.25 & 0.633 & 0.0525 & $0.390_{15}$ \\
1.30 & 0.590 & 0.0725 & $0.271_{10}$ \\
1.35 & 0.545 & 0.0930 & $0.165_{15}$ \\
\hline \multicolumn{5}{c}{$\kappa=1.8$} \\
0.90 & 0.754 & 0.0220 & $0.612_{15}$ \\
0.95 & 0.715 & 0.0313 & $0.475_{15}$ \\
1.00 & 0.672 & 0.0463 & $0.360_{10}$ \\
1.05 & 0.624 & 0.0650 & $0.241_{10}$ \\
1.10 & 0.565 & 0.0880 & $0.140_{10}$ \\
1.13 & 0.530 & 0.1060 & $0.088_{10}$ \\
1.15 & 0.500 & 0.1432 & $0.050_{10}$ \\
\hline
\end{tabular}

TABLE II: Canonical Monte Carlo results for the supercritical pressure, $P$, of the HAY fluid with $\kappa=7$ and 1.8.

\begin{tabular}{c|ccc|ccc}
\hline \hline & \multicolumn{3}{|c|}{$\kappa=7.0$} & \multicolumn{3}{c}{$\kappa=1.8$} \\
\hline$\rho$ & $T=0.432$ & 0.50 & 0.828 & $T=1.0$ & 1.425 & 2.36 \\
\hline 0.2 & 0.052 & 0.077 & 0.188 & 0.130 & 0.193 & 0.487 \\
0.4 & 0.070 & 0.136 & 0.478 & 0.160 & 0.347 & 1.280 \\
0.5 & 0.077 & - & - & 0.210 & 0.530 & 2.040 \\
0.6 & 0.090 & 0.235 & 0.900 & 0.450 & 0.962 & 3.420 \\
0.7 & 0.128 & 0.351 & 1.400 & 1.000 & 1.855 & 5.590 \\
0.8 & 0.240 & 0.580 & 2.200 & 2.400 & 3.631 & 9.450 \\
0.9 & 0.530 & 1.090 & 3.500 & - & - & -
\end{tabular}

TABLE III: Critical parameters of the HCY fluid with different values of the range parameter $\kappa$.

\begin{tabular}{cl|ccccc}
\hline \hline$\kappa$ & Ref. & $T_{c}$ & $\rho_{c}$ & $B_{2}^{*}$ & $P_{c}$ & $Z_{c}$ \\
\hline 0.5 & $\underline{15}$ & 6.958 & 0.260 & -6.474 & - & - \\
1.0 & 15 & 2.460 & 0.280 & -6.290 & - & - \\
1.5 & & 1.451 & 0.304 & -6.277 & 0.128 & 0.300 \\
1.8 & & 1.180 & 0.313 & -6.212 & 0.112 & 0.300 \\
1.8 & $\underline{15}$ & 1.189 & 0.317 & -6.122 & - & - \\
1.8 & $\underline{11}$ & 1.177 & 0.313 & -6.242 & - & - \\
2.0 & $\underline{14}$ & 1.050 & 0.322 & -6.232 & 0.102 & 0.305 \\
2.5 & $\underline{14}$ & 0.840 & 0.336 & -6.206 & 0.086 & 0.303 \\
3.0 & 14 & 0.721 & 0.356 & -6.028 & 0.076 & 0.296 \\
4.0 & 13 & 0.581 & 0.380 & -5.795 & 0.066 & 0.302 \\
5.0 & 13 & 0.500 & 0.393 & -5.684 & 0.060 & 0.305 \\
6.0 & 13 & 0.448 & 0.412 & -5.590 & 0.055 & 0.298 \\
7.0 & $\underline{13}$ & 0.414 & 0.422 & -5.402 & 0.053 & 0.302 \\
\hline
\end{tabular}

Figure Captions

Fig.1 Critical density $\rho_{c}$ as a function of inverse critical temperature $T_{c}^{-1}$ (left panel); critical pressure $P_{c}$ as a function of critical temperature $T_{c}$ (right panel). The results of the HAY fluids are presented by fill circles ( $\mathrm{MC}$ results), and dotted line ( $\left.\mathrm{SCOZA}^{6,15}\right)$. The squares depict Molecular Dynamic simulation data of Ref 15 for $n-6$ potential; dashed line represents SCOZA theory predictions for the square-well fluid 26 .

Fig.2 Reduced vapor-liquid coexistence curves for HAY fluid at different ranges of attractions: $\kappa=7$, and 5 (Ref $\left.{ }^{13}\right) ; \kappa=1.8$ and 1.5 (present work); $\kappa=1$ (Ref ${ }^{15}$ ).

Fig.3 Reduced surface tension $\gamma_{r}$ as a function of reduced temperature $T_{R}$ at different ranges of attraction. The meaning of the symbols is the same as in Fig.2.

Fig.4 Reduced pressure $P_{R}$ as a function of reduced HAY fluid density $\rho_{r}=\rho / \rho_{c}^{2 / 3}$ at three reduced temperatures, $T_{R}=1.042,1.208$, and 2.0. Two ranges of attraction, $\kappa=7$ (squares) and 1.8 (circles) are considered. 


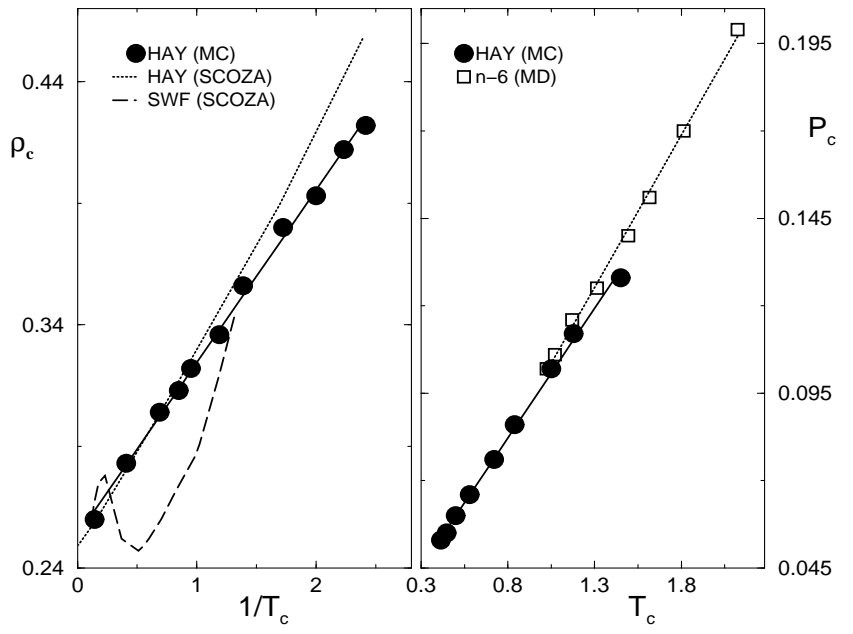

Fig. 1

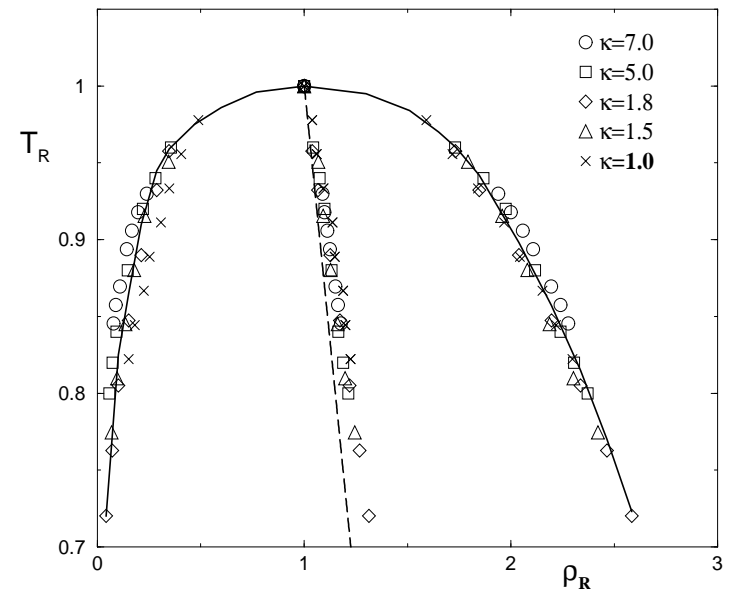

Fig. 2 


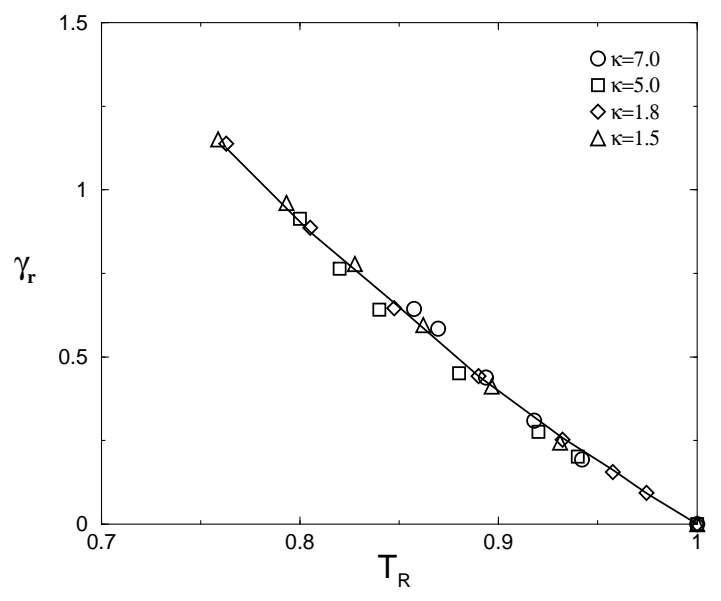

Fig. 3

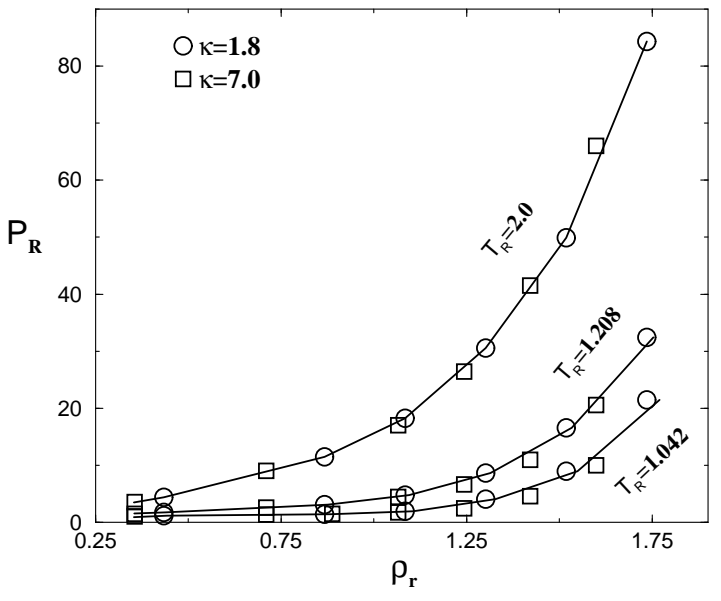

Fig. 4 